\title{
Allergic diseases of the skin and drug allergies - 2013. Longitudinal analysis of fecal microbiota of infants followed-up for eczema till 2 years of age
}

\author{
Gaik Chin Yap ${ }^{1 *}$, Christophe Lay ${ }^{2}$, Marion Aw ${ }^{3}$, Lynette Shek Pei-Chi ${ }^{3}$, Yudong Zhao ${ }^{4}$, Doreen Leow ${ }^{4}$, Bee Wah Lee ${ }^{1}$
}

From 2nd WAO International Scientific Conference (WISC 2012)

Hyderabad, India. 6-9 December 2012

\section{Background}

Studies have suggested that selective microbial targets prevail in the fecal microbiota of infants with eczema. This study aims to evaluate and compare the composition of fecal microbiota of infants who developed eczema by 2 years of age and healthy controls.

\section{Methods}

Children with eczema at 2 years old $(n=26)$ and their matched (for gender, mode of delivery and feeding in first 6 months) healthy controls $(n=26)$ were selected from the placebo group of a cohort of at-risk infants participating in an randomized double-blind placebo controlled trial on the protective effects of supplemental probiotics (first 6 months) on eczema and allergies. Children with eczema were subclassified into atopic eczema $(n=12)$ and non-atopic eczema $(n=14)$. Molecular evaluation of fecal microbiota were conducted using Fluorescence In Situ Hybridization-Flow Cytometry (FISH-FC) for fecal samples collected at 3 days, 1, 3, and 12 months. Probes were selected to target Eubacterium rectale-Clostridium coccoides group (Erec482), Clostridium leptum subgroup (Clep866 and the corresponding competitor probes), Bacteroides-Prevotella group (Bac303), Bifidobacterium genus (Bif164), Atopobium group (Ato291), Lactobacilli- Enterococci group (Lab158), Enterobacteriaceae family (Enter1432) and Clostridium perfringens (Cperf191). Linear mixed model was used to evaluate the longitudinal differences (i.e. 4 time points) of bacterial targets while adjusting for gender, mode of delivery, feeding up to 6 months, and allergic rhinitis and wheezing within the eczema group at 2 years of age.

\section{Results}

Longitudinal analyses over four time points showed that higher relative abundance of Enterobacteriaceae [coefficient (B): 1.104, 95\% confidence interval (CI):0.175-2.033, adj $\mathrm{p}=0.022$ ] in children with eczema by 2 years of age. Similar observations were made when eczema group was subanalyzed into non-atopic and atopic eczema, where higher relative abundance of Enterobacteriaceae [B:1.357, 95\%CI; 0.382-2.332, adj $\mathrm{p}=0.008]$ and [B:1.165, 95\%CI; $0.221-2.109$, adj $\mathrm{p}=0.019$ ] were observed respectively as compared to healthy controls. Relative abundance of Clostridium perfringens were also higher when subanalyzed for non-atopic [B:0.572, 95\%CI; 0.0009-1.144, adj p=0.050] and atopic eczema [B:0.000451, 95\%CI: 0.0001-0.0007, adj $\mathrm{p}=0.012]$ compared to healthy controls.

\section{Conclusions}

Our data suggests that relative abundance of selective microbial targets particularly Enterbacteriaceaea and Clostridium perfringens in the fecal microbiota of infants influence the development of eczema in early childhood.

\section{Author details}

${ }^{1}$ National University of Singapore, Singapore. ${ }^{2}$ Danone Research-Centre for Specialised Nutrition, Singapore. ${ }^{3}$ Paediatrics, National University of Singapore, Singapore. ${ }^{4}$ Singapore Clinical Research Institute, Singapore.

Published: 23 April 2013

doi:10.1186/1939-4551-6-S1-P100

Cite this article as: Yap et al:: Allergic diseases of the skin and drug allergies - 2013. Longitudinal analysis of fecal microbiota of infants followed-up for eczema till 2 years of age. World Allergy Organization Journal 2013 6(Suppl 1):P100.

${ }^{1}$ National University of Singapore, Singapore

Full list of author information is available at the end of the article

(c) 2013 Yap et al; licensee BioMed Central Ltd. This is an Open Access article distributed under the terms of the Creative Commons 